\title{
Brain Characteristics of Individuals Resisting Age-Related Cognitive Decline over Two Decades
}

\author{
Sara Pudas, ${ }^{1,6}$ Jonas Persson, ${ }^{1,2,6}$ Maria Josefsson, ${ }^{3,6}$ Xavier de Luna, ${ }^{3}$ Lars-Göran Nilsson ${ }^{1}$, and Lars Nyberg ${ }^{4,5,6}$ \\ ${ }^{1}$ Department of Psychology, Stockholm University, 10691 Stockholm, Sweden, ${ }^{2}$ Aging Research Center, Karolinska Institute, 11330 Stockholm, Sweden, and \\ ${ }^{3}$ Department of Statistics, Umeå School of Business and Economics, ${ }^{4}$ Department of Integrative Medical Biology (Physiology), ${ }^{5}$ Department of Radiation \\ Sciences (Radiology), and 'Umeå Center for Functional Brain Imaging, Umeå University, 90187 Umeå, Sweden
}

\begin{abstract}
Some elderly appear to resist age-related decline in cognitive functions, but the neural correlates of successful cognitive aging are not well known. Here, older human participants from a longitudinal study were classified as successful or average relative to the mean attritioncorrected cognitive development across $15-20$ years in a population-based sample $(n=1561)$. Fifty-one successful elderly and 51 age-matched average elderly (mean age: 68.8 years) underwent functional magnetic resonance imaging while performing an episodic memory face-name paired-associates task. Successful older participants had higher BOLD signal during encoding than average participants, notably in the bilateral PFC and the left hippocampus (HC). The HC activation of the average, but not the successful, older group was lower than that of a young reference group ( $n=45$, mean age: 35.3 years). HC activation was correlated with task performance, thus likely contributing to the superior memory performance of successful older participants. The frontal BOLD response pattern might reflect individual differences present from young age. Additional analyses confirmed that both the initial cognitive level and the slope of cognitive change across the longitudinal measurement period contributed to the observed group differences in BOLD signal. Further, the differences between the older groups could not be accounted for by differences in brain structure. The current results suggest that one mechanism behind successful cognitive aging might be preservation of $\mathrm{HC}$ function combined with a high frontal responsivity. These findings highlight sources for heterogeneity in cognitive aging and may hold useful information for cognitive intervention studies.
\end{abstract}

\section{Introduction}

Aging is accompanied by an average decline in mental abilities such as processing speed, attention, and episodic memory (Schaie, 1994; Verhaeghen and Salthouse, 1997; Rönnlund et al., 2005). However, there is substantial heterogeneity in cognitive aging trajectories (Christensen et al., 1999; Wilson et al., 2002), with some individuals showing minimal or no decline (Habib et al., 2007). Predictors of successful cognitive aging include high educational attainment, good health, large social network, and lifestyle and genetic factors (Barnes et al., 2007; Habib et al., 2007; Yaffe et al., 2009; Josefsson et al., 2012). However, despite extensive literature on the neural correlates of cognitive decline in aging (Buckner, 2004), the neural bases of successful aging remain unclear.

Successfully aged individuals could have maintained brain integrity (Nyberg et al., 2012), thus being spared from structural and functional alterations of the PFC and MTL that typically

\footnotetext{
Received June 18, 2012; revised March 26, 2013; accepted April 10, 2013.

Author contributions: J.P., L.-G.N., and L.N. designed research; S.P. performed research; M.J. and X.d.L. contributed unpublished reagents/analytic tools; S.P., M.J., and X.d.L. analyzed data; S.P., J.P., and L.N. wrote the paper.

This work was supported by the Swedish Research Council (grants to L.N. and J.P.) and the Knut and Alice Wallenberg Foundation (grant to L.N.). The Betula project is supported by a grant from the Swedish Research Council (to L.N. and L.-G.N.). We thank the staff of the Betula project, Micael Andersson, Alireza Salami, and our collaborators at the Umeå Center for Functional Brain Imaging.

The authors declare no competing financial interests.

Correspondence should be addressed to Sara Pudas, Department of Integrative Medical Biology, Umeå University, 90187 Umeå, Sweden. E-mail: sara.pudas@psychology.su.se.

DOI:10.1523/JNEUROSCI.2900-12.2013

Copyright $\odot 2013$ the authors $\quad 0270-6474 / 13 / 338668-10 \$ 15.00 / 0$
}

accompany age-related cognitive decline (Golomb et al., 1994; Rajah and D'Esposito, 2005; Persson et al., 2012). Successful aging could also be enabled by an innate or acquired resilience to brain pathology, such as cognitive (Stern, 2009) or brain (Satz, 1993 ) reserve. Few neuroimaging studies have explicitly investigated successful aging, but elderly participants have been characterized as cognitively high or low performing. Commonly, greater brain activation in elderly, especially in the PFC, is correlated with higher cognitive performance (Eyler et al., 2011), which has been interpreted as compensatory recruitment to maintain performance. However, contradicting results have been found (Persson et al., 2006) and, for areas such as the MTL, the results are inconsistent (Eyler et al., 2011). Further, consistent with the notion of brain maintenance, some studies instead indicate more youth-like brain activation patterns in high-performing elderly (Waiter et al., 2008; Düzel et al., 2011).

The mixed findings may stem from inadequate or inconsistent definitions of successful aging. Defining successfully aged as the top-performing individuals in an elderly sample is inadequate because it ignores performance levels of younger individuals, and definitions relative to the performance of younger persons might be biased by cohort effects (Rönnlund et al., 2005). Longitudinal designs are advocated for studying both usual aging (Raz and Lindenberger, 2011) and successful aging (Fiocco and Yaffe, 2010). Such designs, however, also face challenges such as selective attrition and psychometric artifacts. The latter include ceiling and floor effects, regression toward the mean artifacts, and mea- 
surement scales with varying sensitivity to change at different levels (Proust-Lima et al., 2007).

Here we addressed some of these issues by defining successful aging relative to the average, attrition-corrected episodic memory development in a large population-based sample $(n=1561$; Nilsson et al., 1997). Successfully aged individuals were identified with a statistical classification method (Little, 1995; Josefsson et al., 2012) and the neural correlates of successful aging were investigated with fMRI in a subsample of elderly $(n=102)$. We focused on areas important for episodic memory and with a documented sensitivity to aging: the MTL and the PFC (Golomb et al., 1994; Rajah and D’Esposito, 2005; Persson et al., 2012).

\section{Materials and Methods}

Participants and selection procedures. The data reported in the present study were derived from three samples from the longitudinal, population-based Betula study (Nilsson et al., 1997). Samples 1 and 3 were recruited at the first and second measurement points, respectively (T1: 1988-1990 and T2: 1993-1995) and formed the basis for the statistical classification model described below. At recruitment, the samples comprised 1000 individuals each, with subjects evenly distributed over 10 age cohorts ranging from 35 to 80 years of age and approximately even gender distributions (full sample characteristics are given by Nilsson et al., 1997). These samples had been followed longitudinally for 15-20 years and cognitively tested every fifth year. In connection with the fifth measurement point, T5 (2008-2010) in Betula, 292 participants from Samples 1 and 3 were scanned with structural and functional MRI. An additional 83 participants from a new sample (sample 6, recruited at T5) were also included in the imaging study, adding up to 375 persons in total. Participation in the imaging sample was randomly offered to participants who had completed cognitive testing at T5 until a pre-allotted number of slots were filled, which were stratified by age and gender.

The final groups, on which the imaging analyses were based, are presented in the Results section. The successful aging group consisted of all elderly participants in the imaging sample who had been classified as cognitive maintainers in our previous study (Josefsson et al., 2012; classification procedures described below) and did not meet the exclusion criteria described in the next paragraph. The control group consisted of participants who were classified as having average cognitive development over 15-20 years, and these were age-matched person by person to the included successful agers. Selection of participants from the imaging sample into the average control group was based on the shortest standardized Euclidian distance to the average baseline cognitive test score and the average slope of cognitive change. This metric was calculated for each age cohort in the full Betula sample (1954 participant baseline scores and the slopes of 1561 participants with two or more measurements). This procedure ensured that the most representative participants (i.e., those closest to the average baseline score and slope) from the imaging sample were selected into the control group. Whenever a participant with the shortest Euclidian distance met any of the exclusion criteria, the person with the next shortest distance was selected instead. In addition to the two elderly groups, fMRI data from a young reference group were used to interpret the group differences between the successful and average elderly. This group included data from all participants in the imaging sample who did not meet exclusion criteria and were 45 years of age or younger.

Before imaging analyses, 24 successful agers were excluded for the following reasons: problems with visual acuity $(n=4)$, poor-quality structural T1 image preventing satisfactory normalization (e.g., outlier status, or missing data; $n=6$ ), not fulfilling preestablished performance criteria for the scanner task $(>42 \%$ correct responses and $<50 \%$ missing responses; $n=9$ ), and health-related issues or remarks from the radiologist screening the structural scans for abnormities $(n=5)$. Further, 29 participants classified as having average cognitive development were omitted from the average control group for the following reasons: not reaching performance criteria $(n=26)$, problems with visual acuity $(n=$ $2)$, and misunderstanding the scanner task $(n=1)$. An additional average participant was excluded after preliminary imaging analyses due to out- lier status across all voxels/clusters. The young group was subjected to the same exclusion criteria as the older groups, but only one young participant had to be excluded due to a technical problem with response collection.

All included participants were in good general health, without major neurological impairments or diagnoses, and had normal, or corrected to normal, vision. Participants signed a written informed consent form and were financially compensated for their participation. The study was approved by the local ethics board at Umeå University.

Cognitive measures and classification of cognitive change. Cognitive change was assessed with a composite of five episodic memory scores, measured at 5-year intervals during 20 years of the Betula study (Nilsson et al., 1997). The composite consisted of: (1) immediate free recall of 16 imperative verb-noun sentences that were enacted by the participant, (2) delayed cued recall of nouns from the previously enacted sentences, (3) immediate free recall of 16 verbally and visually presented verb-noun sentences, (4) delayed cued recall of nouns from the previously presented sentences, and (5) immediate free recall of 12 verbally presented nouns. Testing procedures remained constant across measurement occasions and full details have been given previously (Nilsson et al., 1997).

The statistical classification procedures have previously been described in full detail (Josefsson et al., 2012) and encompassed baseline memory scores of all 1954 participants in Samples 1 and 3 from the Betula study and the slopes of 1561 participants with two or more measurement points. The slopes (i.e., linear rates of cognitive change over 15-20 years) were computed for each participant through ordinary leastsquares regression of the episodic memory composite on time. The slopes and baseline scores were then entered into a random-effects pattern-mixture model (Little, 1995) to estimate an attritioncorrected average memory development in each of 10 age cohorts in our full sample.

Significant negative correlations between baseline level and rate of change in our cognitive scores were observed for every age cohort (with $r$ ranging from -0.22 to -0.43 ) in the Betula samples. Individuals starting with high levels of performance tended to display more decline, whereas individuals with low initial performance seemed to improve or decline less rapidly. Rather than being indicative of true biological processes, these associations likely reflect a combination of statistical and psychometric artifacts, such as regression toward the mean or functional floor and ceiling effects (Salthouse, 2012), or that the tests have varying sensitivity to change depending on the level of the scale (Proust-Lima et al., 2007). For the purposes of our classification, this entails that a zero or positive slope alone is not an adequate definition of successful aging, because that would lead to an overrepresentation of individuals with apparent improvement from a low level in the successful aging group. Therefore, we wanted to define a successful ager as a person with a moderate/high initial memory score and a better than average slope for a given baseline score.

Therefore, each participant was classified based on how his/her initial baseline memory score and estimated rate of change compared with the average for his/her age cohort as estimated from the pattern-mixture model. To obtain an outcome measure that accounted for both initial level and slope of change in memory scores, we used the predicted final score as a cutoff measure because the predicted final score, by definition, is a linear combination of the baseline score plus rate of change multiplied by time in the study (i.e., 15 or 20 years). This allowed us to consider cognitive performance across the entire 15-20 years that the participants had been followed in our definition of successful aging. All individuals with predicted final scores greater than 1 SD from the estimated average score in each respective age cohort were classified as successful agers (denoted as "maintainers" in Josefsson et al., 2012). Individuals with final scores below 1 SD from the average were classified as decliners and excluded from the present study. The remaining participants were classified as average and formed the basis for the average control group in the imaging analyses. The reason for not including the decliners in subsequent analyses was that only seven decliners remained in our imaging sample after applying the exclusion criteria. These individuals were also, on average, 8.1 years younger than the successful agers in the sample. 
In the current application of our statistical classification model, and in contrast to our previous study (Josefsson et al., 2012), separate models were estimated for each sample (831 persons in Sample 1, and 730 in Sample 3) because the samples had different numbers of measurement points.

In-scanner memory task. Participants performed a face-name pairedassociates task (Kauppi et al., 2011; Persson et al., 2011; Salami et al., 2012b) implemented in E-prime software (Psychology Software Tools). This task comprised of blocks of encoding and retrieval and an active baseline task involving simple perceptual discrimination (e.g., subjects had to press a button when the fixation cross was replaced by a circle). Encoding stimuli were photographs of unfamiliar faces presented separately, together with a common first name. In the retrieval blocks, the previously presented faces reappeared together with three letters. Participants were instructed to indicate the correct first letter of the name by pressing one of three buttons. If unsure, they were instructed to guess. Presentation time was $4 \mathrm{~s}$ per face in both encoding and retrieval conditions, with 1.5-4.5 s randomized interstimulus intervals (allowing for both event-related and blocked analyses). The mean duration between encoding and retrieval of a given face was $85.1 \mathrm{~s}(\mathrm{SD}=26.1)$. A total of 24 face-name pairs were presented throughout the task, which lasted $\sim 10$ min. Block and stimulus order was pseudorandomized and constant across all participants. Before scanning, participants were familiarized with the task by completing a short practice version of it.

The scanner task was presented to the participants on a computer screen, seen through a tilted mirror on the head coil. Responses were collected on a scanner-compatible response pad. Participants were given headphones and earplugs to dampen scanner noise, and cushions inside the head coil minimized head movements.

MRI acquisition. Structural and functional imaging was performed on a 3 tesla General Electric scanner equipped with a 32 channel head coil. Functional data were acquired with a gradient echoplanar imaging sequence ( 37 transaxial slices, thickness: $3.4 \mathrm{~mm}$, gap: $0.5 \mathrm{~mm}$, TR $2000 \mathrm{~ms}$, TE $30 \mathrm{~ms}$, flip angle $80^{\circ}$, field of view $25 \times 25 \mathrm{~cm}$ ). To allow for progressive saturation of the fMRI signal, 10 dummy scans were collected and discarded before experimental image acquisition. High-resolution T1weighted structural images were collected with a 3D fast spoiled gradient echo sequence (180 slices with a $1 \mathrm{~mm}$ thickness, TR $8.2 \mathrm{~ms}$, TE $3.2 \mathrm{~ms}$, flip angle $12^{\circ}$, field of view $25 \times 25 \mathrm{~cm}$ ). Finally, white matter integrity was assessed with a diffusion tensor imaging (DTI) T2-weighted spinechoplanar sequence (64 slices, TR $8000 \mathrm{~ms}$, TE $84.4 \mathrm{~ms}$, flip angle $90^{\circ}$, field of view $25 \times 25 \mathrm{~cm}, b=1000 \mathrm{~s} / \mathrm{mm}^{2}$; six B0 images were collected). Images were acquired in three repetitions, with 32 independent directions.

Data preprocessing and analysis. Preprocessing and analysis of imaging data were done in SPM8 (Wellcome Department of Imaging Science, Functional Imaging Laboratory, http://www.fil.ion.ucl.ac.uk/spm) implemented in MATLAB 7.11 (MathWorks). Batching of analyses, visualization, and extraction of median parameter estimates across clusters were performed with software developed inhouse (DataZ).

Functional data. The fMRI data were preprocessed to correct for differences in within-volume slice acquisition times and head movement (by realignment and unwarping). The functional images were coregistered to each participant's structural T1 image. First-order statistical analyses were set up, using voxelwise general linear models with the experimental conditions (i.e., encoding, retrieval, and baseline) as regressors, modeled as boxcars, and convolved with the standard hemodynamic response function. An event-related control analysis was also performed for the encoding condition, including only subsequently remembered items. For this analysis, trials were categorized based on responses in the retrieval condition. Separate regressors were constructed for items that were later recalled and items that were forgotten. Each trial was treated as an impulse, and these events were convolved with the standard hemodynamic response function. Linear contrasts were performed to generate SPM maps representing the differences in brain activation between subsequently recalled items compared with the baseline. The statistical models contained the six realignment parameters as covariates of no interest. The resulting contrast images were then nonlinearly normalized to a local, age-representative template (see below). Finally, the images were affinely aligned to the MNI standard space and smoothed with an $8 \mathrm{~mm}$ FWHM Gaussian kernel. All reported coordinates are in MNI space.

Second-level analyses were set up with random-effects models to display the main effects of the tasks across all 147 participants (i.e., encoding-baseline and retrieval-baseline) using one-sample $t$ tests. These were corrected for multiple comparisons using the FWE rate at $p<0.05$. Two-sample $t$ tests were used to test group differences between successful and average older participants in the encoding-baseline and retrievalbaseline contrasts. An uncorrected statistical threshold of $p<0.001$ and an extent threshold of 10 contiguous voxels were used for these contrasts. Significant clusters were localized with automated anatomical labeling (Tzourio-Mazoyer et al., 2002). Finally, for ROI analyses, brain-behavior correlations, and visualization of results, median BOLD effect sizes were extracted across significant clusters and across a hippocampus (HC) mask generated from our sample template with FreeSurfer software (http://surfer.nmr.mgh.harvard.edu). Voxel-level BOLD effects were calculated using the formula $\left(\left[\beta_{\text {Encoding }}-\beta_{\text {Baseline }}\right] / \beta_{\text {Constant }}\right) \times 100$, where the $\beta$ s are the regression coefficients for the encoding and baseline experimental conditions from the first-level analyses and $\beta_{\text {Constant }}$ is the mean intensity across the session. Values were then averaged across the extent of the cluster for each participant and entered into SPSS statistics software for testing. For the ROI analyses, a Bonferroni-corrected statistical threshold was used, which was based on the number of clusters (i.e., 5: $0.05 / 5=0.01)$. SPSS software was also used to test differences in behavioral data and background variables (one-way unrelated ANOVAs with Dunnett's T3 method for multiple comparisons, independent sample $t$ tests and Pearson's correlations).

Creation of sample-specific template. The template was created from high-resolution T1 images of 292 healthy individuals (51\% female) ages $25-81$ years (mean, $60.5 \pm 13.1)$ selected from the imaging cohort $(n=$ 375 in total). Participants were omitted from the template if they did not meet performance criteria on the scanner task $(n=63)$, did not perform the scanner task $(n=10)$, had reported health problems or visible brain pathologies $(n=2)$, or were structural outliers $(n=8)$. Structural outliers were defined by the "Sample Homogeneity" function in the voxelbased morphometry (VBM) toolbox for SPM8. The T1 images of the selected participants were segmented (using the "New Segment" option in SPM8), and the resulting gray and white matter partitions averaged to a mean template using the DARTEL toolbox (Ashburner, 2007). Subjectspecific deformation parameters to the mean template were then computed and the inverse of these applied to each participant's structural image. These warped images were again averaged to a new mean template and the procedure was iterated six times in total, resulting in a final sample-representative template.

Multimodal imaging analyses. The coregistered structural T1-weighted images were preprocessed with the VBM toolbox in SPM8. They were segmented (with the "New Segment" option), normalized to the samplespecific template, and smoothed with an $8 \mathrm{~mm}$ FWHM Gaussian kernel. After modulation, in which individual volumetric information was reintroduced after normalization by multiplying with the Jacobian determinants, the images were entered as covariates of no interest in an ANCOVA model in the biological parametric mapping (BPM) toolbox (Casanova et al., 2007), with the fMRI data as the primary modality. A separate preprocessing was performed on the functional data (following the above-described procedures), specifying the voxel size to match the resolution of the structural data. BPM employs the same general linear model approach as SPM, but allows each voxel to have a different design matrix based on each participant's gray matter voxel values. The results from such an analysis can be interpreted as reflecting group differences in brain activation that cannot be accounted for by differences in local gray matter volume. The results were thresholded at $p<0.001$ (uncorrected).

DTI data. The DTI-weighted data were preprocessed and subjected to a tract-based analysis using the University of Oxford's Center for Functional Magnetic Resonance Imaging of the Brain (FMRIB) software library (Wakana et al., 2003) package (http://www.fmrib.ox.ac.uk/fsl), specifically tract-based spatial statistics (Smith et al., 2006). Exact procedures were described previously (Salami et al., 2012a). In brief, mean fractional anisotropy (FA) values for 12 white matter tracts were ex- 


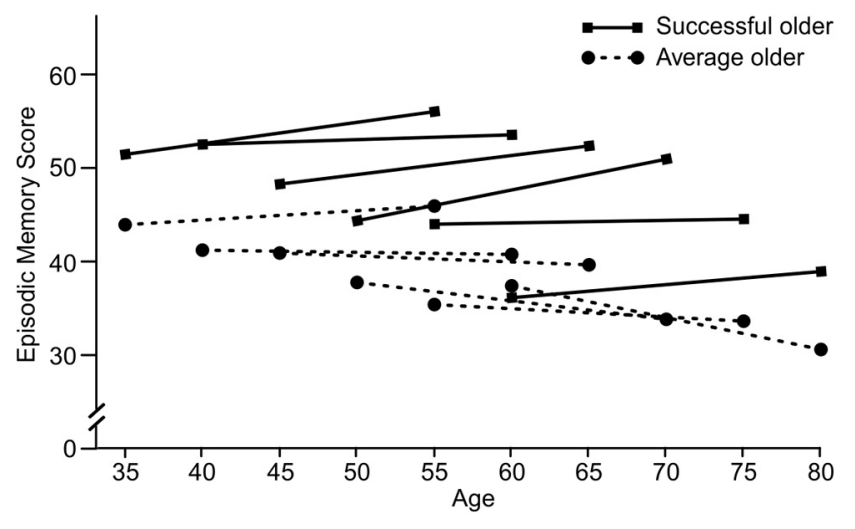

Figure 1. Longitudinal memory change. Average memory slopes for each age cohort in the successful and average older groups. $n=6-16$ for age cohorts $55-75$ years of age at last measurement; the 80-year-old cohorts contained one average and three successful older participants. Episodic memory scores represent a composite of five episodic memory tasks (maximum $=76$ ).

tracted for each participant by averaging across the length of the tract and across hemispheres. Tracts were defined according to JHU ICBMDTI-81 white matter labels, which are included in the FMRIB software library atlas tools (Wakana et al., 2003), and the following were included in the analyses: genu, body, and splenium of the corpus callosum, cingulum, corona radiata, corticospinal tract, external and internal capsule, superior/inferior fronto-occipital fasciculus, superior longitudinal fasciculus, sagittal striatum, and the uncinate fasciculus. Group differences between the successful and average older participants were tested in SPSS statistics software using a multivariate ANOVA. Due to poor image quality, two participants were excluded from DTI analyses (one successful and one average participant).

\section{Results}

\section{Classification outcome}

The application of the statistical classification method on the longitudinal Betula Samples 1 and $3(n=1561)$ resulted in $22 \%$ successful agers, 67\% average individuals and 11\% decliners, which approximates the percentages reported in our previous study (Josefsson et al., 2012). In the subset of individuals that comprised our imaging sample ( $n=292$ from Samples 1 and 3), the proportion of successful agers was $25 \%$; $7 \%$ were classed as decliners and the remaining $68 \%$ had average cognitive development. The slight differences might reflect the fact that the imaging sample was more selected, although it is important to remember that the imaging participants were defined in relation to the full population-based samples.

After the exclusion procedures and selection of age-matched controls (described in Materials and Methods), the resulting groups consisted of 51 successful agers (mean age, $68.8 \pm 7.1$ years) and 51 average-classed elderly. The cognitive development across 20 years for each age cohort within the successful and average groups can be seen in Figure 1. Note that, consistent with previous longitudinal reports (Schaie, 1994; Rönnlund et al., 2005), the expected age-related memory decline in the average group only becomes evident after the age of 60-65 years.

Group-level cognitive variables are shown in Table 1, which additionally displays demographics and scores for the young reference group. Overall, the two older groups differed significantly on both initial memory performance and slope of memory change over time (Table 1). Note that the difference in slope was observed despite the wide within-group age range and the inclusion of individuals younger than 65 years, who are not expected to have experienced age-related cognitive decline. In these younger age groups, successful individuals mainly differed from average individuals by having a more positive rate of change (Fig. 1 ). The positive slopes are likely driven by retest effects that are typical for longitudinal datasets (Rönnlund et al., 2005). Therefore, the values of the slopes reported here should not be interpreted literally. Nevertheless, the slope for the average group, -0.084 , was very close to the mean attrition-corrected slope for all individuals in the full population-based sample: -0.078 (agecohorts, $65-70$ years, $n=361$ ). This confirms the representativeness of the individuals selected into the average control group. Overall, there was a significant improvement in cognitive scores across time for the successful group $\left(t_{(50)}=4.94, p<0.001\right.$, paired samples $t$ test), whereas the average group displayed a significant decrease $\left(t_{(50)}=2.41, p<0.05\right)$. In addition, note that the lack of group differences between successful and average individuals on a global cognitive screening measure, the MiniMental State Examination (Folstein et al., 1975), indicates that the average group by no means was cognitively impaired despite the decline in episodic memory performance.

\section{Scanner task performance}

As can be seen in Table 1, task accuracy of successful agers was slightly higher than that of average agers, although the difference was not significant. There was no difference in reaction times (RTs). Young participants performed better than both older groups both in terms of accuracy and RTs (all statistics are reported in Table 1).

\section{Functional imaging}

Across all 147 participants, similar to what was found previously for other subsamples (Kauppi et al., 2011; Salami et al., 2012b), the face-name task engaged a widespread network of brain areas typical for episodic memory tasks, including the occipital, parietal, frontal, and temporal areas and the cerebellum (for encoding- and retrieval-related activations, see Fig. 2). In addition, there was robust activation of bilateral $\mathrm{HC}$ in all three groups (for encoding-related HC activation, see Fig. 3A). Contrasting the successful and the average older participants for episodic encoding relative to baseline showed that successful agers had stronger activation in several regions (Table 2), notably in the left PFC and the left HC. No regions showed significantly higher activation in average participants compared with successful agers. For the retrieval-baseline contrast, no areas were found in which successful agers displayed increased activation relative to average controls. The reverse retrieval contrast (average $>$ successful) produced one significant cluster $\left(280 \mathrm{~mm}^{3}\right)$ in the ventral/medial PFC (BA 11; peak $x, y, z=8,32,-30 ; t=4.15$, $p<0.001)$. This effect was driven by higher baseline-related activation in the successful relative to the average older group and will not be discussed further. No differences in either direction were seen in the MTL during retrieval.

To facilitate interpretation of the observed differences between the successful and average agers, their encoding-related activation (median parameter estimates) across the left $\mathrm{HC}$ and PFC clusters was extracted and compared with the activation of a young reference group. First, in the left HC, young participants had a comparable degree of activation to that of successful older subjects, but they differed from average older subjects $\left(F_{(1,94)}=\right.$ $8.08, p<0.005$; Fig. $3 B$ ). This indicates that successful agers had preserved $\mathrm{HC}$ activation in this cluster relative to young participants, whereas the average older individuals failed to reach the activation levels of the young group. Second, across the four PFC clusters (Fig. 4), the response of the successful agers was compa- 
Table 1. Participant demographics and cognitive characteristics

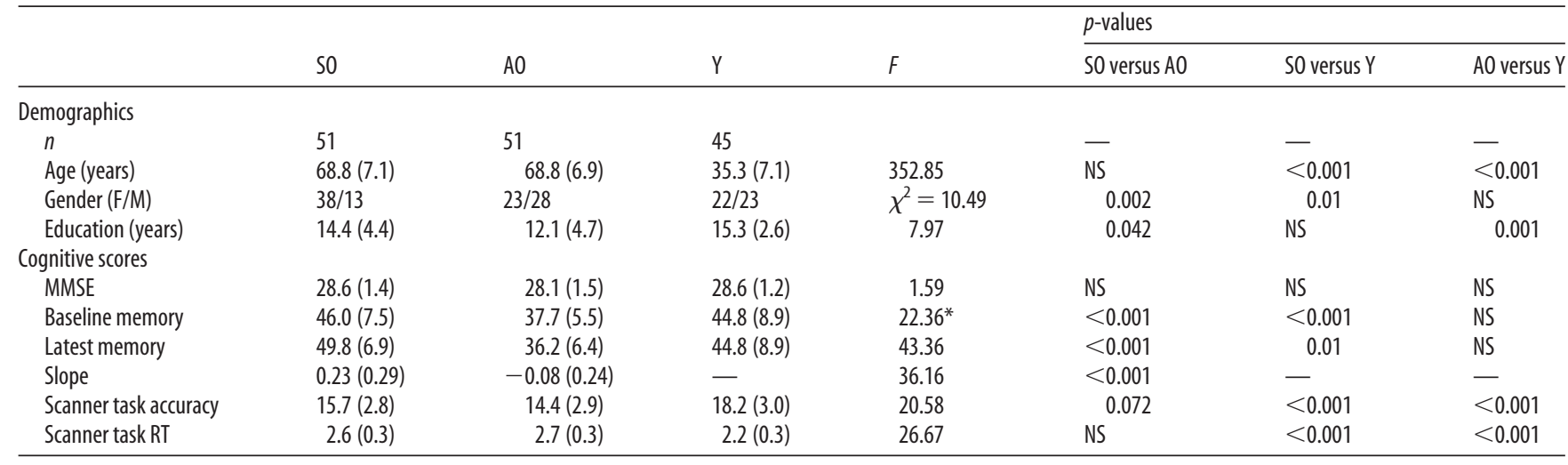

Figures are group means with SDs shown in parentheses. Baseline memory was measured in 1988-1995 for the older groups and in 2008-2010 for the younger groups. Latest memory score and MMSE were measured approximately 9 months before scanning. Baseline and latest memory scores of the young were identical due to only one measurement. Slope represents linear change in memory scores over $15-20$ years.

SO indicates successful older; A0, average older; Y, young; MMSE, Mini Mental State Examination; NS, not significant.

*Controlling for age when entering the study.

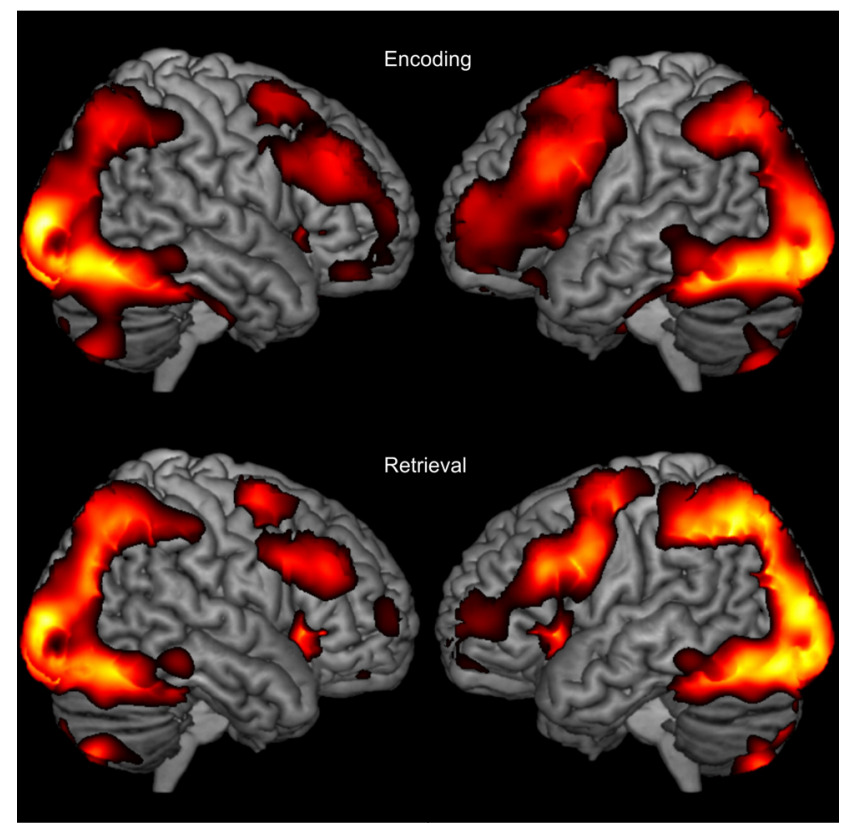

Figure 2. Main effects of encoding and retrieval. Shown are brain areas that were engaged during episodic encoding and retrieval, respectively, compared with the baseline task, across all three groups ( $n=147)$. Results are displayed at a FWE-corrected threshold of $p<0.05$.

rable to that of young participants in three of the clusters (Fig. 4, clusters $b-d)$ and significantly higher in the left inferior frontal cluster $\left(F_{(1,94)}=11.32, p<0.001\right.$; Fig. 4 , cluster a). Comparing average older with young participants, the group difference did not reach Bonferroni-corrected significance levels in any peak (although $p=$ 0.012 for cluster c, Fig. 4). Therefore, the results for the frontal cortex were more mixed, but in none of the clusters did the activation levels of successful agers show an age-related decrease relative to the young group.

Due to inequalities in gender proportions and educational attainment across groups (Table 1), BOLD responses from the $\mathrm{HC}$ and frontal clusters were also examined in relation to possible effects of gender and education. Across all 83 female and 64 male subjects included in the analyses, there was no significant gender effect on $\mathrm{HC}$ or PFC activations (all $p>0.10$ ). Education was significantly correlated with activation in the HC only $(r=0.18$, $p<0.05)$. However, this effect did not remain significant when controlling for age (partial correlation; $r=0.14, p=0.10$ ). We also replicated the results in the two left PFC clusters, the ACC, and the left $\mathrm{HC}$ when rerunning the encoding contrast between successful and average older participants but only including items that were subsequently correctly remembered (Fig. 5). Therefore, the results were not primarily driven by differences in scanner task accuracy.

\section{Brain-behavior correlations}

Next, scanner task accuracy and mean RT were correlated with degree of activation across all 102 participants in the two older groups to investigate whether brain activation contributed significantly to memory performance. Of our five ROIs, there was a significant correlation with task accuracy only in the left HC cluster $(r=0.33, p<0.001$; all four frontal clusters, $p>0.10)$. The correlation was comparable in size in both groups, but only significant in the average older group (average older: $r=0.32$, $p<0.05$; successful older: $r=0.25, p=0.079$ ). No significant correlations between RT and brain activation were observed within or across groups (all $p>0.10$ ), indicating the absence of time-on-task effects in these areas. We also investigated the relation between chronological age and brain activation across both groups, which revealed a significant negative correlation in the left $\mathrm{HC}(r=-0.32, p<0.05$; all frontal clusters, $p>0.10)$. Again, the correlations were comparable in magnitude but only reached significance in the average older group $(r=-0.32, p<0.05$; successful older: $r=-0.21, p=0.143)$.

\section{Structural imaging}

We next investigated whether the brain activation differences between the successful and average older groups could be driven by differences in brain anatomy, specifically loss of structural integrity in the average group. Using BPM, in which structural T1-weighted images are included as covariates in the functional analyses in a voxel-by-voxel manner, we replicated the group differences in the PFC and HC. This result indicated that the functional differences were not driven by smaller gray matter volumes in the average participants. Instead, the BPM analysis resulted in a larger extent of the left HC cluster (from 240 to 667 $\mathrm{mm}^{3}$ ) and revealed two additional clusters in the right MTL (right parahippocampal gyrus: peak $x, y, z=31,-16,-23, t=$ 3.69; right HC: $x, y, z=25,-10,16$; $t=3.38$ ), in which successful agers had increased activation relative to average agers. The cause of this difference was investigated post hoc by extraction of gray 
A

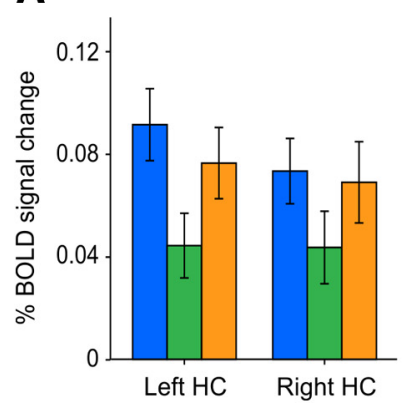

B

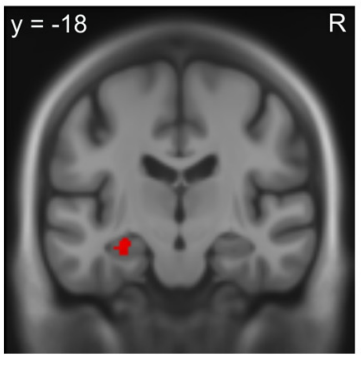

$\square$ Successful older Average older $\square$ Young
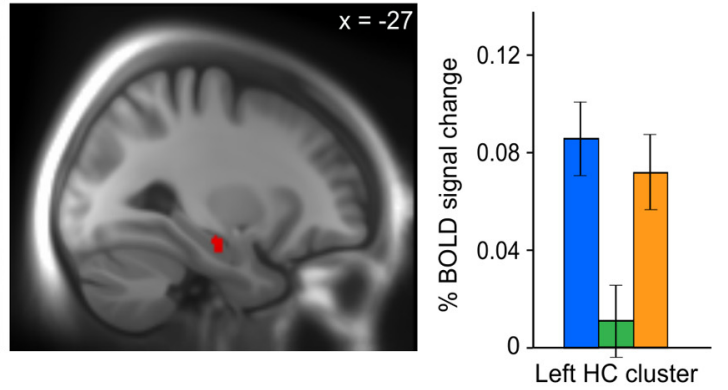

Figure 3. Group differences in $\mathrm{HC}$ recruitment during encoding. Shown are the HCBOLD signal changes from the encoding-baseline contrast for the entire $H C$ formation $(A)$ and the left $H C$ cluster from the group contrast between successful and average older participants $(\boldsymbol{B})$. The HC activation in $\boldsymbol{A}$ was calculated within left and right $\mathrm{HC}$ masks generated with FreeSurfer software. $\boldsymbol{B}$ displays a coronal slice at $y=-18$ and a sagittal slice at $x=-27$. Error bars represent \pm 1 SEM.

Table 2. Brain regions more recruited by the successful agers compared with average agers during episodic encoding

\begin{tabular}{|c|c|c|c|c|c|c|c|}
\hline Side & Region & BA & $x$ & $y$ & $z$ & $\begin{array}{l}\text { Volume } \\
\left(\mathrm{mm}^{3}\right)\end{array}$ & $t$ \\
\hline Right & Cerebellum & & 30 & -72 & -36 & 376 & 3.89 \\
\hline Left & Inferior frontal gyrus & 45 & -42 & 34 & 4 & 1368 & 3.81 \\
\hline Right & Occipital & $18 / 19$ & 42 & -88 & 8 & 81 & 3.80 \\
\hline Left & Inferior frontal gyrus & 44 & -50 & 10 & 30 & 648 & 3.78 \\
\hline Right & Cerebellum & & 2 & -76 & -34 & 224 & 3.76 \\
\hline Left & $\mathrm{HC}$ & & -30 & -14 & -16 & 240 & 3.58 \\
\hline Right & Anterior cingulate & 32 & 10 & 30 & 30 & 120 & 3.57 \\
\hline Right & Supplementary motor area & 6 & 4 & 14 & 68 & 80 & 3.56 \\
\hline Right & Inferior frontal gyrus & 45 & 54 & 32 & 20 & 80 & 3.47 \\
\hline
\end{tabular}

Coordinates are in MNI space.

matter volume values from the segmented and normalized structural images using the MTL clusters from the BPM analyses as masks. Successful agers were found to have smaller gray matter volumes in these areas than average participants (Table 3), but for the clusters in left HC and right parahippocampal gyrus, the difference did not remain significant after adding gender into the statistical model ( $p=0.281$ and 0.168 , respectively). For the right $\mathrm{HC}$ cluster in the BPM analyses, the group difference in volume remained after controlling for gender $\left(F_{(1,98)}=4.46, p<0.05\right)$. We also investigated group differences in white matter integrity measured with DTI across 12 white matter tracts specified in the Materials and Methods section. There were no significant differences in average FA values between the two older groups, as assessed with a multivariate ANOVA (including gender as a factor), Pillai's trace $\left(F_{(12,85)}=1.41, p=0.176\right)$. Group-level FA values are given in Table 4 .

\section{Separating the contributions of level and slope}

Because our successful and average aging groups differed both on initial level and change in cognitive scores, it could be argued that the observed group differences in BOLD signal could have been driven mainly by the difference in initial level. We therefore performed two sets of additional analyses to clarify the contribution of initial level and slope to the observed group differences in brain activation. First, we selected subgroups of successful and average elderly participants matched on initial level. The age range of these subgroups was restricted to 65 years and older, because this is the age range during which memory decline can be expected. The resulting groups comprised 25 individuals each, with the successful agers being slightly, but not significantly, older ( 72.7 vs
70.5 years; $\left.t_{(48)}=1.68 ; p=0.1\right)$. The groups did not differ in initial memory level (successful older: $40.0, \mathrm{SD}=5.0$; average older: $\left.39.6, \mathrm{SD}=2.6 ; t_{(48)}=0.28, p=0.78\right)$. They did, however, differ on slope of memory change (successful older: $0.33, \mathrm{SD}=$ 0.30 ; average older: $\left.-0.21, \mathrm{SD}=0.20), t_{(48)}=7.5, p<0.001\right)$. Longitudinal cognitive scores can be seen in Figure $6 A$. Figure $6 B$ displays group differences in encoding-related BOLD signal in the clusters of interest from the original analysis. As can be seen from the figure, the subgroups differed significantly in all clusters (with $t$ ranging from 2.4 to 2.9 , all $p<0.05$ ). On a whole-brain level, at $p<0.005$ (uncorrected), there was also a significant HC cluster overlapping with the cluster from the original analysis (Fig. 6C). In addition, the remaining successful older participants who were initially high performing ( $n=13,65$ years or older) did not differ from the initially lower-performing successful individuals in any cluster (all $p>0.4$ ). However, the high-performing successful group did differ from the average individuals with high initial levels in three of the clusters (HC, anterior cingulate, and right PFC, $p \leq 0.05$ ), which approached significance in the larger left PFC cluster $(-42,34,4 ; p=0.083)$.

Second, we performed a set of hierarchical regression analyses on the individual BOLD values to investigate whether both the slope and initial level of cognitive performance contributed to brain activation in each of the clusters observed in our main analysis. All participants across the successful and average older groups were included in these analyses $(n=102)$. Because chronological age was correlated with initial level of cognitive performance $(r=-0.52, p<0.001)$, we controlled for age in these regressions by entering it before initial level and slope. Among the 102 participants included in our imaging analyses, there was no correlation between slope and initial level $(r=0.0, p=0.997)$, in contrast to our full sample of 1561 individuals. In the regression analyses, we entered initial level before slope to investigate whether slope explained a significant proportion of the variance after accounting for initial level. The results indicated that for the left HC cluster, although age accounted for a significant proportion of the variance $\left(R^{2}=0.06, p<0.05\right)$, the initial level failed to reach significance when entered after age $\left(R^{2}\right.$ change $=0.024$, $p=0.108)$. Slope, however, did explain a significant proportion of the remaining variance in $\mathrm{HC}$ BOLD signal $\left(R^{2}\right.$ change $=$ $0.048, p<0.05)$. The same pattern of results emerged for the anterior cingulate cluster (except for age not reaching significance, $p=0.087$; initial level $R^{2}$ change $=0.024, p=0.117$; slope $R^{2}$ change $\left.=0.062, p<0.05\right)$. For all of the three lateral PFC 

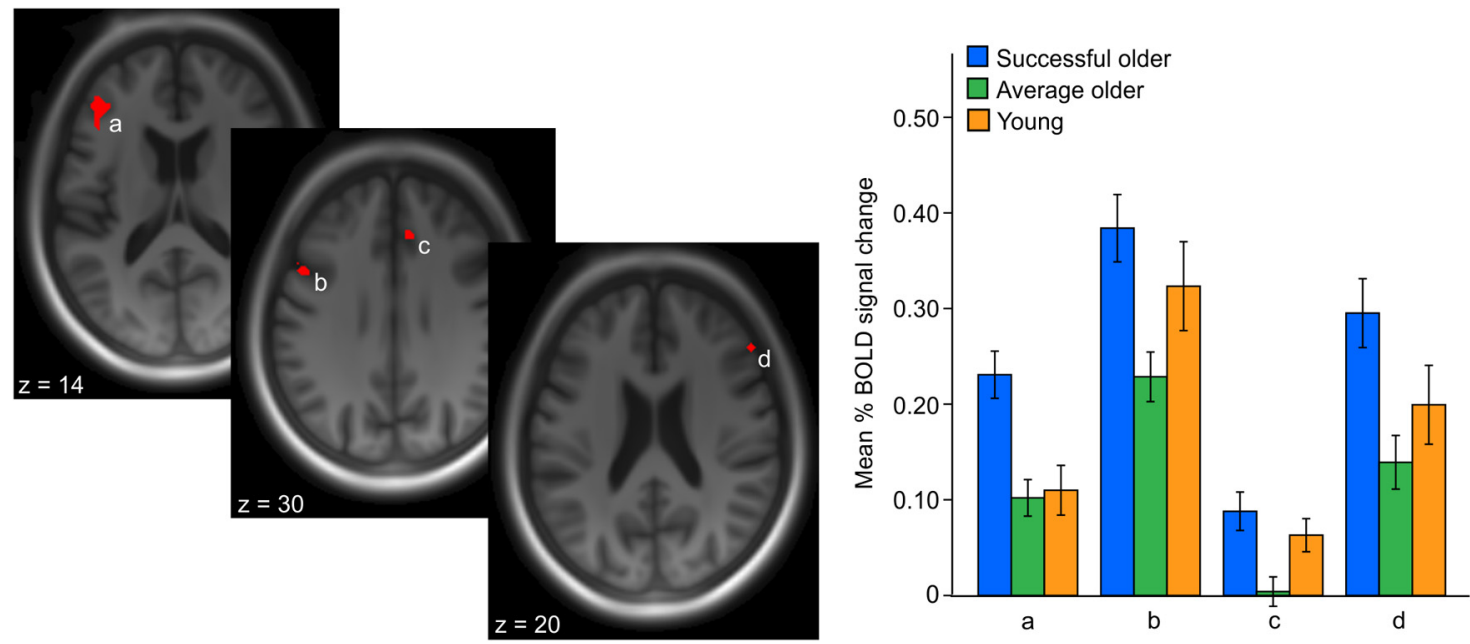

Figure 4. Group differences in frontal recruitment during encoding. Clusters a and b were located in left inferior frontal gyrus, cluster c in the anterior cingulate cortex, and cluster $d$ in right inferior frontal gyrus (peak voxels, a: $-42,34,4 ; b$ : $-50,10,30 ; c: 10,30,30 ; d: 54,32,20$ ). Error bars represent \pm 1 SEM.
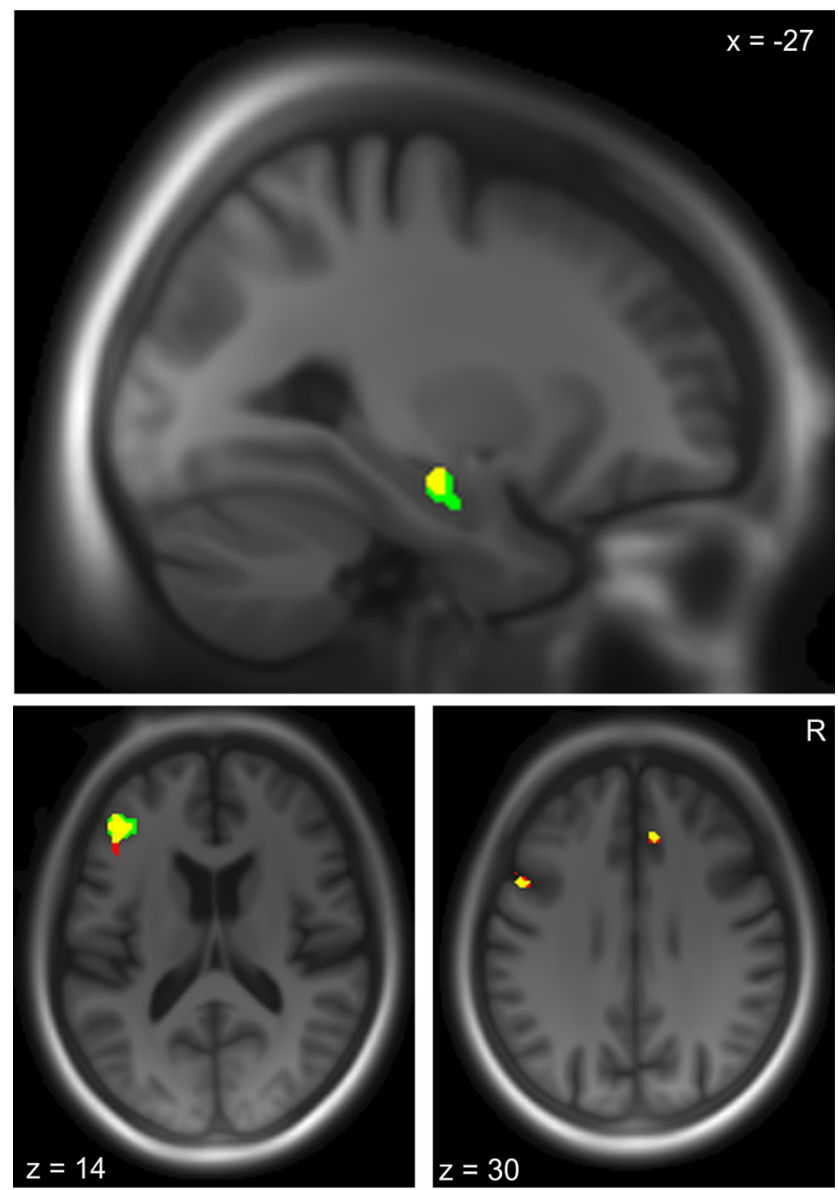

Figure 5. Replication of findings when only considering correctly recalled items. Sagittal (top) and transverse (bottom) slices depicting overlap between the original encoding contrast for successful above average older participants (in red), overlaid with an event-related analysis of the same contrast only using subsequently correctly remembered items (green). Yellow areas show overlap between the analyses, notably two clusters in the left PFC, the anterior cingulate cortex, and the left HC. Both contrasts are displayed at the same statistical significance level ( $p=0.001$, uncorrected).

clusters, both slope and initial level significantly predicted BOLD signal variance (left PFC $-42,34$, 4: initial level $R^{2}$ change $=$ $0.078, p<0.005$, slope $R^{2}$ change $=0.047 p<0.05$; left PFC -50 , 10, 30: initial level $R^{2}$ change $=0.059, p<0.05$, slope $R^{2}$
Table 3. Mean gray matter volume

\begin{tabular}{lccc}
\hline Cluster & Successful older & Average older & Cluster size \\
\hline Right parahippocampal gyrus & $48.5(4.7)$ & $50.7(5.3)$ & 92 \\
Right HC & $15.9(1.6)$ & $17.0(2.0)$ & 27 \\
Left HC & $384.9(33.2)$ & $397.4(41.3)$ & 667 \\
\hline
\end{tabular}

Data are expressed in cubic millimeters with SDs shown in parentheses. Cluster size denotes the total size of the cluster in the functional analyses. The values for each group are the proportion of gray matter within the total cluster.

Table 4. Mean FA values with SDs in parentheses

\begin{tabular}{lll}
\hline White matter tract & Successful older & Average older \\
\hline Corpus callosum, genu & $0.635(0.034)$ & $0.650(0.027)$ \\
Corpus callosum, body & $0.642(0.037)$ & $0.650(0.036)$ \\
Corpus callosum, splenium & $0.785(0.022)$ & $0.794(0.028)$ \\
Cingulum & $0.556(0.038)$ & $0.565(0.031)$ \\
Corona radiata & $0.452(0.029)$ & $0.461(0.026)$ \\
Corticospinal tract & $0.590(0.027)$ & $0.597(0.032)$ \\
External capsule & $0.453(0.026)$ & $0.459(0.028)$ \\
Internal capsule & $0.590(0.024)$ & $0.602(0.022)$ \\
Superior/inferior fronto-occipital fasciculi & $0.522(0.033)$ & $0.533(0.025)$ \\
Superior longitudinal fasciculus & $0.486(0.026)$ & $0.494(0.023)$ \\
Sagittal striatum & $0.526(0.033)$ & $0.538(0.025)$ \\
Uncinate fasciculus & $0.516(0.042)$ & $0.533(0.036)$ \\
\hline
\end{tabular}

change $=0.039, p<0.05$; right PFC 54, 32, 20: initial level $R^{2}$ change $=0.042, p<0.05$, slope $R^{2}$ change $=0.042, p<0.05$ ) Therefore, these analyses indicate that it is mainly differences in slope that account for BOLD signal variance in the $\mathrm{HC}$ and anterior cingulate clusters identified in the main analyses of successful and average older adults. For the lateral frontal clusters, both initial level and slope accounted for significant proportions of the variance in BOLD signal.

\section{Discussion}

We used a classification procedure (Josefsson et al., 2012 based on Little, 1995) to identify successfully aged individuals in a longitudinal, population-based sample $(n=1561)$ followed for 15-20 years. Correcting for selective attrition by pattern-mixture modeling (Little, 1995) allowed us to define successful aging relative to the average cognitive development in each age cohort of our sample. Great care was taken in formulating the definition so that cognitive performance across the entire longitudinal measurement period was taken into account and that psychometric 
A

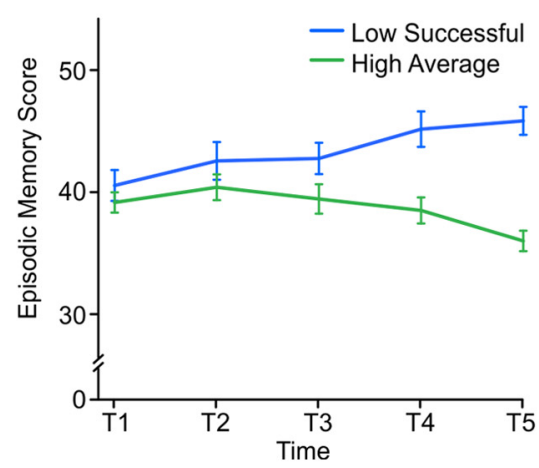

B

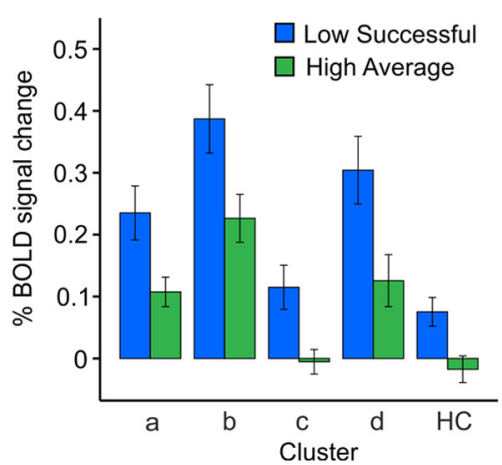

C

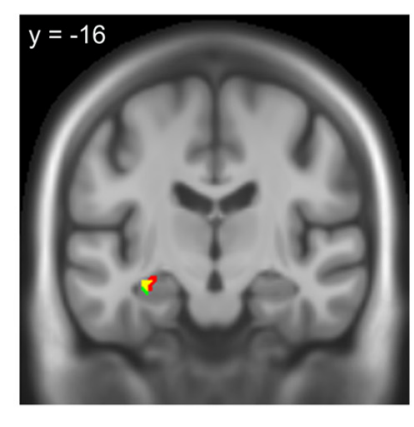

Figure 6. Subgroups matched on initial memory level. $\boldsymbol{A}$, Average longitudinal memory scores for successful and average older subgroups matched on initial memory level ( $n=25$ per group). $\boldsymbol{B}$, Mean BOLD signal change for the matched subgroups for the clusters of interest identified in the encoding-baseline contrast in the full groups. Clusters a-d reflect frontal clusters from Figure 4 (peak voxels, a: $-42,34,4 ; \mathrm{b}$ : $-50,10,30 ; \mathrm{c}: 10,30,30$; $\mathrm{d}: 54,32,20$ ), whereas $\mathrm{HC}$ reflects the HC cluster in Figure 2 B (peak voxel: $-30,-14,-16$ ). C, 0 verlap (in yellow) between HC clusters from the full group contrast (in red) and a whole-brain contrast of the matched subgroups (thresholded at $p=0.005$, in green).

artifacts did not induce bias. The resulting successful aging group had on average a higher initial level of cognitive performance, as well as a more positive trajectory over time, than individuals classified as average. The higher than average initial level is partially a consequence of the way we defined our groups, but it has previously been suggested that those with high initial cognitive ability might experience less cognitive decline in aging (Richards et al., 2004, but see also Gow et al., 2012). However, because of the statistical tendency for higher-performing individuals to decline in our dataset, we are not in a strong position to answer the question of whether age is kinder to the initially more able (Thompson, 1954). However, we are confident that, because our successful individuals resisted the general tendency for highperformers to decline, they are truly successfully aged. In addition, our longitudinal data allowed us to consider potential differential effects of initial level and change in cognitive performance on brain activation, which are at risk of being confounded in cross-sectional studies.

Regarding the brain characteristics of successful aging, our results showed that maintenance of high episodic memory performance across 15-20 years was related to higher HC and frontal activation during episodic memory encoding, than for agematched controls with a typical trajectory of age-related memory change. In a subregion of the left anterior HC, successful agers had activation levels comparable to those of young individuals, whereas the average older participants had significantly lower activation than young participants. This pattern suggests that successful agers are spared from, or have suffered less, age-related reduction of $\mathrm{HC}$ function. The observed group differences are not likely to be driven solely by preexisting differences in $\mathrm{HC}$ function or memory performance from younger age, because the brain activation differences remained when matching subgroups on initial level of memory. In addition, our regression analyses indicated that only slope of cognitive change, not initial level, explained a significant proportion of variation in HC BOLD signal. Given the importance of the MTL for episodic memory (Squire et al., 2004), this finding of HC preservation likely accounts, at least partially, for the resistance of the successful aging group to age-related memory decline.

Reduced HC activation is commonly found in lowperforming elderly (Daselaar et al., 2003; Persson et al., 2012), but it has not been established that the successfully aged are spared from such changes. Our findings converge with a recent study characterizing successful aging as having a brain activation pattern similar to young individuals (Düzel et al., 2011). However, our study is the first to show HC preservation using a definition based on longitudinal cognitive change. These results also support a recent proposal that maintaining youth-like brain characteristics is one mechanism behind successful cognitive aging (Nyberg et al., 2012). Furthermore, our findings suggest that, even in those elderly with the most typical trajectory of cognitive change, without substantial loss, there is some reduction in $\mathrm{HC}$ function. Findings regarding $\mathrm{HC}$ function in aging have been inconsistent (Eyler et al., 2011) and sometimes no reduction in HC function is seen in elderly samples (Persson et al., 2011). In light of our findings, the latter type of outcome may stem from a study sample biased toward successfully aged, and/or not including very old individuals (Salami et al., 2012b). This underscores the importance of carefully characterizing the study sample with regard to cognitive status in aging studies, and especially the importance of including longitudinal measurements of cognitive function.

Regarding the frontal activation differences, our findings replicate and extend previous cross-sectional studies showing more pronounced PFC activation in high-performing than in lowperforming older individuals during episodic memory tasks (Cabeza et al., 2002; Rosen et al., 2002) and cognitive tasks in general (Eyler et al., 2011). Commonly, higher PFC activation in high-performing elderly has been interpreted as compensatory (Cabeza et al., 2002; Reuter-Lorenz and Cappell, 2008; Eyler et al., 2011), having arisen in response to unfavorable age-related brain-cognition changes (Greenwood, 2007). Although this interpretation cannot be ruled out, lack of frontal activation-performance and activation-age correlations in our data makes it precarious. Compensatory activation would be expected to correlate with age, assuming that more detrimental changes that require more compensation are acquired with age. An alternative explanation is that successful agers have had a better frontal function since youth. This would be consistent with a longitudinal imaging study demonstrating that apparent cross-sectional agerelated increases in frontal activation may be driven by highperforming elderly, who actually display activation decreases when followed longitudinally (Nyberg et al., 2010).

Although the frontal activation differences might not reflect compensation per se, a high frontal responsivity, as found in our successful agers, could reflect a higher neural capacity (Stern, 
2009), and might contribute to the preservation of cognitive abilities during the course of aging. In general, neural capacity denotes a capability to recruit more neural resources to cope with a challenging task, and is hypothesized to be a manifestation of cognitive reserve (Stern, 2009). Interestingly, cognitive reserve is commonly associated with high educational attainment (Stern, 2009), as was also found in our successful agers (Table 1). Our previous study identified a number of additional significant predictors of being classified as a successful ager in relation to our full population-based sample (Josefsson et al., 2012). These included female gender, living together with someone, being physically active, and being a carrier of the met-allele of the COMT gene. These factors are known to be associated with beneficial effects on cognitive and brain function (Fratiglioni et al., 2004; de Frias et al., 2004; Hillman et al., 2008; Andreano and Cahill, 2009; Krach et al., 2010), which further validates our definition of successful aging. Our results thus suggest that many factors, some of which are amendable, may contribute to successful cognitive aging and that these manifest neurally as high $\mathrm{HC}$ and prefrontal function.

Previous successful aging studies have compared successful individuals with those with decline on global cognitive screening tests (Yaffe et al., 2009; Rosano et al., 2012), but our study contrasted successfully aged with the most average performers on more sensitive measures of episodic memory ability. Despite this tight comparison, our findings were robust and could not be explained by differences in structural brain integrity, gender, education, or differences in scanner task performance. Further, our results were not solely driven by differences in initial level of memory performance, as demonstrated by first replicating the group differences in frontal and HC BOLD response in subgroups matched on initial memory level. Second, regression analyses on the individual BOLD values converged to show that cognitive change also contributed significantly to the observed group differences. Third, splitting the successful group by initial level of performance revealed a similar response profile in initially higher- and lower-performing successful individuals relative to an initially high-performing average subgroup. These observations demonstrate that both a high initial level and a more positive than average slope of cognitive change contribute to the successful aging brain phenotype found here. Our definition of successful aging did not include maintenance of low levels of performance, however. It thus remains to be elucidated whether our results also generalize to individuals with such a pattern. Furthermore, in binary classification procedures like the current one, misclassification of individuals is possible. However, the large sample size should have minimized the influence of such validity threats on the overall results. Future studies with longitudinal imaging data will be needed to shed light on the causal mechanisms behind the differential brain activation pattern observed here.

In conclusion, the present results are noteworthy for several reasons. First, they highlight the importance of preserved $\mathrm{HC}$ and high frontal function for the maintenance of good memory function in aging. By including longitudinal measures of cognitive change in our definition of successful cognitive aging, these results substantiate and extend previous findings (Cabeza et al., 2002; Düzel et al., 2011; Eyler et al., 2011). Further, the current results complement our previous longitudinal imaging findings that implicate $\mathrm{HC}$ dysfunction in cognitive decline (Persson et al., 2012). This strongly suggests that HC function is a key source of heterogeneity in cognitive aging trajectories. The findings also help to delineate the boundaries of what constitutes typical cognitive aging, and therefore have general significance for the cog- nitive neuroscience of aging. Finally, the study of successful aging in itself is an important complement to the abundant literature on age-related cognitive decline. Identifying predictors and neural correlates of successful cognitive aging might take us one step closer toward tackling the increased societal burden of an aging population.

\section{References}

Andreano JM, Cahill L (2009) Sex influences on the neurobiology of learning and memory. Learn Mem 16:248-266. CrossRef Medline

Ashburner J (2007) A fast diffeomorphic image registration algorithm. Neuroimage 38:95-113. CrossRef Medline

Barnes DE, Cauley JA, Lui LY, Fink HA, McCulloch C, Stone KL, Yaffe K (2007) Women who maintain optimal cognitive function into old age. J Am Geriatr Soc 55:259-264. CrossRef Medline

Buckner RL (2004) Memory and executive function in aging and AD: multiple factors that cause decline and reserve factors that compensate. Neuron 44:195-208. CrossRef Medline

Cabeza R, Anderson ND, Locantore JK, McIntosh AR (2002) Aging gracefully: compensatory brain activity in high-performing older adults. Neuroimage 17:1394-1402. CrossRef Medline

Casanova R, Srikanth R, Baer A, Laurienti PJ, Burdette JH, Hayasaka S, Flowers L, Wood F, Maldjian JA (2007) Biological parametric mapping: A statistical toolbox for multimodality brain image analysis. Neuroimage 34:137-143. CrossRef Medline

Christensen H, Mackinnon AJ, Korten AE, Jorm AF, Henderson AS, Jacomb P, Rodgers B (1999) An analysis of diversity in the cognitive performance of elderly community dwellers: individual differences in change scores as a function of age. Psychol Aging 14:365-379. CrossRef Medline

Daselaar SM, Veltman DJ, Rombouts ARB, Raaijmakers JG, Jonker C (2003) Neuroanatomical correlates of episodic encoding and retrieval in young and elderly subjects. Brain 126:43-56. CrossRef Medline

de Frias CM, Annerbrink K, Westberg L, Eriksson E, Adolfsson R, Nilsson LG (2004) COMT Gene Polymorphism Is Associated with Declarative Memory in Adulthood and Old Age. Behav Genet 34:533-539. CrossRef Medline

Düzel E, Schütze H, Yonelinas AP, Heinze HJ (2011) Functional phenotyping of successful aging in long-term memory: preserved performance in the absence of neural compensation. Hippocampus 21:803-814. CrossRef Medline

Eyler LT, Sherzai A, Kaup AR, Jeste DV (2011) A review of functional brain imaging correlates of successful cognitive aging. Biol psychiatry 70:115122. CrossRef Medline

Fiocco AJ, Yaffe K (2010) Defining successful aging: the importance of including cognitive function over time. Arch Neurol 67:876-880. CrossRef Medline

Folstein MF, Folstein SE, McHugh PR (1975) "Mini-mental state": A practical method for grading the cognitive state of patients for the clinician. J Psychiatr Res 12:189-198. CrossRef Medline

Fratiglioni L, Paillard-Borg S, Winblad B (2004) An active and socially integrated lifestyle in late life might protect against dementia. Lancet Neurol 3:343-353. CrossRef Medline

Golomb J, Kluger A, de Leon MJ, Ferris SH, Convit A, Mittelman MS, Cohen J, Rusinek H, De Santi S, George AE (1994) Hippocampal formation size in normal human aging: a correlate of delayed secondary memory performance. Learn Mem 1:45-54. CrossRef Medline

Gow AJ, Johnson W, Mishra G, Richards M, Kuh D, Deary IJ; HALCyon Study Team (2012) Is age kinder to the initially more able?: Yes, and no. Intelligence 40:49-59. CrossRef

Greenwood PM (2007) Functional plasticity in cognitive aging: review and hypothesis. Neuropsychology 21:657-673. CrossRef Medline

Habib R, Nyberg L, Nilsson LG (2007) Cognitive and non-cognitive factors contributing to the longitudinal identification of successful older adults in the Betula study. Neuropsychol Dev Cogn B Aging Neuropsychol Cogn 14:257-273. CrossRef Medline

Hillman CH, Erickson KI, Kramer AF (2008) Be smart, exercise your heart: exercise effects on brain and cognition. Nat Rev Neurosci 9:58-65. CrossRef Medline

Josefsson M, de Luna X, Pudas S, Nilsson LG, Nyberg L (2012) Genetic and lifestyle predictors of 15-year longitudinal change in episodic memory. J Am Geriatr Soc 60:2308-2312. CrossRef Medline

Kauppi K, Nilsson LG, Adolfsson R, Eriksson E, Nyberg L (2011) KIBRA 
polymorphism is related to enhanced memory and elevated hippocampal processing. J Neurosci 31:14218-14222. CrossRef Medline

Krach S, Jansen A, Krug A, Markov V, Thimm M, Sheldrick AJ, Eggermann T, Zerres K, Stöcker T, Shah NJ, Kircher T (2010) COMT genotype and its role on hippocampal-prefrontal regions in declarative memory. Neuroimage 53:978-984. CrossRef Medline

Little RJ (1995) Modeling the drop-out mechanism in repeated-measures studies. J Am Stat Assoc 90:1112-1121. CrossRef

Nilsson LG, Bäckkman L, Erngrund K, Nyberg L, Adolfsson R, Bucht G, Karlsson S, Widing M, Winblad B (1997) The Betula prospective cohort study: memory, health, and aging. Neuropsychol Dev Cogn B Aging Neuropsychol Cogn 4:1-32. CrossRef

Nyberg L, Salami A, Andersson M, Eriksson J, Kalpouzos G, Kauppi K, Lind J, Pudas S, Persson J, Nilsson LG (2010) Longitudinal evidence for diminished frontal cortex function in aging. Proc Natl Acad Sci U S A 107: 22682-22686. CrossRef Medline

Nyberg L, Lövdén M, Riklund K, Lindenberger U, Bäckman L (2012) Memory, aging and brain maintenance. Trends Cogn Sci 16:292-305. CrossRef Medline

Persson J, Nyberg L, Lind J, Larsson A, Nilsson LG, Ingvar M, Buckner RL (2006) Structure-function correlates of cognitive decline in aging. Cereb Cortex 16:907-915. CrossRef Medline

Persson J, Kalpouzos G, Nilsson LG, Ryberg M, Nyberg L (2011) Preserved hippocampus activation in normal aging as revealed by fMRI. Hippocampus 21:753-766. CrossRef Medline

Persson J, Pudas S, Lind J, Kauppi K, Nilsson LG, Nyberg L (2012) Longitudinal structure-function correlates in elderly reveal MTL dysfunction with cognitive decline. Cereb Cortex 22:2297-2304. CrossRef Medline

Proust-Lima C, Amieva H, Dartigues JF, Jacqmin-Gadda H (2007) Sensitivity of four psychometric tests to measure cognitive changes in brain agingpopulation-based studies. Am J Epidemiol 165:344-350. CrossRef Medline

Rajah MN, D’Esposito M (2005) Region-specific changes in prefrontal function with age: a review of PET and fMRI studies on working and episodic memory. Brain 128:1964-1983. CrossRef Medline

Raz N, Lindenberger U (2011) Only time will tell: cross-sectional studies offer no solution to the age-brain-cognition triangle: comment on Salthouse. Psychol Bull 137:790-795. CrossRef Medline

Reuter-Lorenz PA, Cappell KA (2008) Neurocognitive aging and the compensation hypothesis. Curr Dir Psychol Sci 17:177-182. CrossRef

Richards M, Shipley B, Fuhrer R, Wadsworth ME (2004) Cognitive ability in childhood and cognitive decline in mid-life: longitudinal birth cohort study. BMJ 328:552-557. CrossRef Medline

Rönnlund M, Nyberg L, Bäckman L, Nilsson LG (2005) Stability, growth, and decline in adult life span development of declarative memory: crosssectional and longitudinal data from a population-based study. Psychol Aging 20:3-18. CrossRef Medline

Rosano C, Aizenstein HJ, Newman AB, Venkatraman V, Harris T, Ding J, Satterfield S, Yaffe K; Health ABC Study (2012) Neuroimaging differences between older adults with maintained versus declining cognition over a 10-year period. Neuroimage 62:307-313. CrossRef Medline

Rosen AC, Prull MW, O’Hara R, Race EA, Desmond JE, Glover GH, Yesavage
JA, Gabrieli JD (2002) Variable effects of aging on frontal lobe contributions to memory. Neuroreport 13:2425-2428. CrossRef Medline

Salami A, Eriksson J, Nilsson LG, Nyberg L (2012a) Age-related white matter microstructural differences partly mediate age-related decline in processing speed but not cognition. Biochim Biophys Acta 1822:408-415. CrossRef Medline

Salami A, Eriksson J, Nyberg L (2012b) Opposing effects of aging on largescale brain systems for memory encoding and cognitive control. J Neurosci 32:10749-10757. CrossRef Medline

Salthouse TA (2012) Does the direction and magnitude of cognitive change depend on initial level of ability? Intelligence 40:352-361. CrossRef Medline

Satz P (1993) Brain reserve capacity on symptom onset after brain injury: a formulation and review of evidence for threshold theory. Neuropsychology 7:273-295. CrossRef

Schaie KW (1994) The course of adult intellectual development. Am Psychol 49:304-313. CrossRef Medline

Smith SM, Jenkinson M, Johansen-Berg H, Rueckert D, Nichols TE, Mackay CE, Watkins KE, Ciccarelli O, Cader MZ, Matthews PM, Behrens TE (2006) Tract-based spatial statistics: voxelwise analysis of multi-subject diffusion data. Neuroimage 31:1487-1505. CrossRef Medline

Squire LR, Stark CE, Clark RE (2004) The medial temporal lobe. Annu Rev Neurosci 27:279-306. CrossRef Medline

Stern Y (2009) Cognitive reserve. Neuropsychologia 47:2015-2028. CrossRef Medline

Thompson DE (1954) Is age kinder to the initially more able? Proc Iowa Acad Sci 61:439-441.

Tzourio-Mazoyer N, Landeau B, Papathanassiou D, Crivello F, Etard O, Delcroix N, Mazoyer B, Joliot M (2002) Automated anatomical labeling of activations in SPM using a macroscopic anatomical parcellation of the MNI MRI single-subject brain. Neuroimage 15:273-289. CrossRef Medline

Verhaeghen P, Salthouse TA (1997) Meta-analyses of age-cognition relations in adulthood: estimates of linear and nonlinear age effects and structural models. Psychol Bull 122:231-249. CrossRef Medline

Waiter GD, Fox HC, Murray AD, Starr JM, Staff RT, Bourne VJ, Whalley LJ, Deary IJ (2008) Is retaining the youthful functional anatomy underlying speed of information processing a signature of successful cognitive ageing? An event-related fMRI study of inspection time performance. Neuroimage 41:581-595. CrossRef Medline

Wakana S, Jiang H, Nagae-Poetscher LM, van Zijl PC, Mori S (2003) Fiber tract-based atlas of human white matter anatomy. Radiology 230:77-87. CrossRef Medline

Wilson RS, Beckett LA, Barnes LL, Schneider JA., Bach J, Evans DA, Bennett DA (2002) Individual differences in rates of change in cognitive abilities of older persons. Psychol Aging 17:179-193. CrossRef Medline

Yaffe K, Fiocco AJ, Lindquist K, Vittinghoff E, Simonsick EM, Newman AB, Satterfield S, Rosano C, Rubin SM, Ayonayon HN, Harris TB; Health ABC Study (2009) Predictors of maintaining cognitive function in older adults: the Health ABC study. Neurology 72:2029-2035. CrossRef Medline 